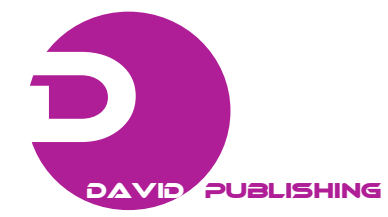

\title{
Nonlinear Control Model of Synchronous Motor with Excitation and Damper Windings
}

\author{
Marijo Šundrica ${ }^{1}$ and Zlatko Maljković ${ }^{2}$ \\ 1. Electric Power Generation Department, KONČAR_Power Plant and Electric Traction Engineering Inc., Zagreb 10000, Croatia \\ 2. Dep. of Electric Machines, Drives and Automation, Faculty of Electrical Engineering and Computing, Zagreb 10000, Croatia
}

Received: July 09, 2013 / Accepted: September 09, 2013 / Published: February 28, 2014.

\begin{abstract}
In this paper, a new control method for synchronous motor with excitation and damper windings is presented. It is based on one type of nonlinear control; feedback linearization control. To make a realization in the sense of electric drive, symmetrical space vector PWM (pulse width modulation) is applied. Estimation of damper winding currents via Lyapunov function for the whole estimated system is done. The aim of control is to make tracking system for rotor speed and square of stator flux. Simulation of motor starting to predefined operating points is done, and also maintaining these points during step change of load torque is obtained. Simulations give good results.
\end{abstract}

Key words: Synchronous motor, nonlinear control, Lyapunov stability, observer control, feedback linearization, electric drive simulation.

\section{Introduction}

This paper is organized as follows: At first there is an introduction to synchronous machine and its control; after that synchronous machine model, the state observer and control law are introduced; then internal dynamics and system stability are analyzed, control scheme is given, and modulation is defined; there is a case study with simulation results; and finally contribution, future work and conclusion are given.

\subsection{Synchronous Machine Application}

SM (synchronous machine) with excitation and damper windings is used in power systems mainly for two reasons: first and more frequent for power generation and second as electric drive. Here, the main interest is to check SM variable speed operation.

Usual AC drive system, for any type of machine, consists of frequency convertor that ensures soft

Corresponding author: Marijo Šundrica, developing designer, Ph.D. student, research fields: electric machines modeling and control. E-mail: marijo.sundrica@koncar-ket.hr. operation through the whole speed range. Electric drive of SM due to some of its characteristics (high efficiency, the possibility of overloading, ease of maintenance, etc.) finds particular application in practice: coal mines, metal and cement industries, ship propulsion, conveyor belts and large compressors, etc..

In power generation units, there is also need for SM to work in variable speed operation: for operation of windmills with SM generators; and for starting, especially in pumped-storage hydroelectricity.

\subsection{Vector Control}

At the beginning, the control of AC motors started as scalar. As induction motors started to replace DC motors, idea of AC motor control according to control of DC motors appeared. That idea later became known as vector control in which decoupling between torque and flux control was to be achieved. Due to the complexity of AC motor dynamics it is possible only if the flux is constant.

Nevertheless, a great number of vector control asynchronous AC drives have been designed, but not 
so many SM vector control drives. It is not only because of the greater number of asynchronous motors but also because of the complexity of SM system dynamics. There are some researches about SM vector control, but they all give palliative results; they have very complex control structures and limited control domains [1, 2] mainly because of the fact that in SM toque component of stator current essentially changes magnetic flux.

\subsection{Nonlinear Control}

Because of the salient poles, a large number of coupled variables and high nonlinearity, SM is a complex dynamic system.

There are many academic attempts to upgrade well known scalar and vector control models using nonlinear control systems. Based on mathematical stability proof, nonlinear control improves performance of a control system and sometimes gives some new control possibilities.

Recent literature gives a certain type of nonlinear control for SM: backstepping method [3] in which damper winding currents were not considered, the method of passivity [4] which failed to give better results, as well as some others $[5,6]$ in which decoupling has not been considered. Literature generally brings only mathematical models of control without adequate simulational implementation of the entire power system. Because of this, application of a new nonlinear method for SM and its implementation is an open research field and also a challenging task.

Since the SM includes damping windings whose currents usually can not be measured, control is often associated with an estimator (observer).

In this case, the observer is also in the function of control; so, it is necessary to ensure the convergence of the whole system "observer + controller" to get globally asymptotic stable system. Literature gives some examples of such systems [7-10] but none for this kind of motor.

\section{Synchronous Motor Model}

SM dynamic system which is based on magnetic fluxes as state variables is not suitable; it is therefore necessary to express the system through the currents as state variables:

$$
\left\{\begin{array}{c}
i_{d} \\
i_{f} \\
i_{D} \\
i_{q} \\
i_{Q} \\
\dot{\omega}
\end{array}\right\}=\left\{\begin{array}{c}
a_{1} i_{d}+a_{2} i_{q} \omega+a_{3} i_{Q} \omega+a_{4} i_{f}+a_{5} i_{D}+a_{6} u_{d}+a_{7} u_{f} \\
b_{1} i_{d}+b_{2} i_{q} \omega+b_{3} i_{Q} \omega+b_{4} i_{f}+b_{5} i_{D}+b_{6} u_{d}+b_{7} u_{f} \\
c_{1} i_{d}+c_{2} i_{q} \omega+c_{3} i_{Q} \omega+c_{4} i_{f}+c_{5} i_{D}+c_{6} u_{d}+c_{7} u_{f} \\
d_{1} i_{q}+d_{2} i_{d} \omega+d_{3} i_{f} \omega+d_{4} i_{D} \omega+d_{5} i_{Q}+d_{6} u_{q} \\
f_{1} i_{q}+f_{2} i_{d} \omega+f_{3} i_{f} \omega+f_{4} i_{D} \omega+f_{5} i_{Q}+f_{6} u_{q} \\
j_{1} i_{d} i_{q}+j_{2} i_{f} i_{q}+j_{3} i_{q} i_{D}+j_{4} i_{d} i_{Q}+j_{5} M_{T}
\end{array}\right\}(1)
$$

Coefficients $a_{1}, a_{2}$, etc. incorporate synchronous machine standard parameters: $L_{m d}, L_{m q}, r_{s}, r_{f}$, etc..

Parameters' (resistance andinductance) variation due to time and nonlinearity of magnetic circuits was not considered.

\section{State Observer}

According to Eq. (1), Luenberger full order type of observer is used; the estimated system is given in Eq. (2):

Errors are defined as:

$$
\begin{aligned}
& e_{1}=i_{d}-\widehat{\iota_{d}} ; e_{2}=i_{f}-\widehat{\iota_{f}} ; e_{3}=i_{D}-\widehat{\iota_{D}} ; \\
& e_{4}=i_{q}-\widehat{\iota_{q}} ; e_{5}=i_{Q}-\widehat{\iota_{Q}} ; e_{6}=\omega-\widehat{\omega} .
\end{aligned}
$$

Coefficients $k_{11}, k_{12}$, etc. will be used to achieve the convergence.

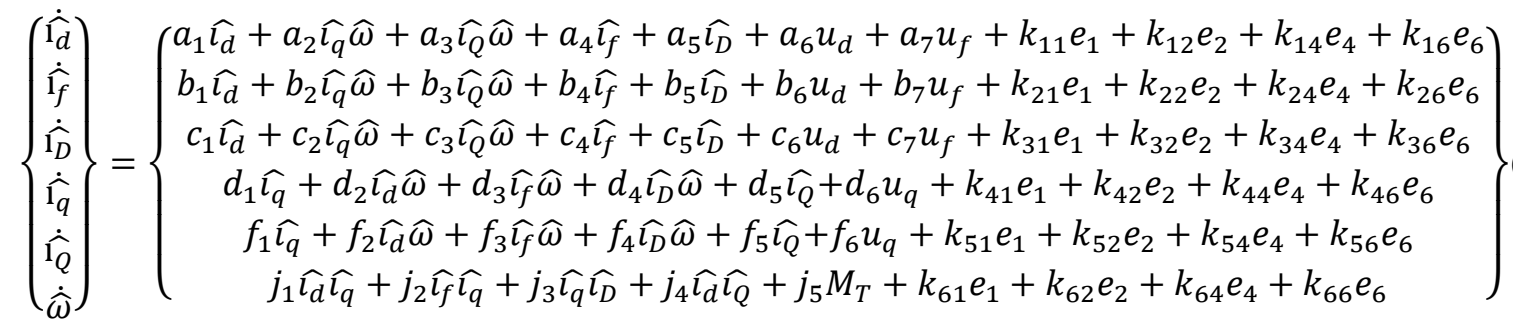

Error dynamics is defined as Eqs. (1) and (2).

Error system stability will be proved by Lyapunov; the following Lyapunov function is defined:

$$
V_{1}=\frac{e_{1}^{2}}{2}+\frac{e_{2}^{2}}{2}+\frac{e_{4}^{2}}{2}+\frac{e_{6}^{2}}{2}+\frac{1}{2}\left(k_{1} e_{3}+k_{2} e_{5}\right)^{2}+k_{3} \frac{e_{5}^{2}}{2}
$$

According to Lyapunov, stability is achieved if differential of $V_{l}$ is negative for any value of the error. 
Calculating error dynamics (1)-(2) with appropriate coefficients, differential of Eq. (3) can always be made negative for the entire speed range of the motor.

\section{Control Law}

\subsection{Feedback Linearization}

Control based on feedback linearization method. As far as it is known, feedback linearization has not been applied to SM before. On the other hand, this method enables cancelation of all system nonlinearities (and linear parts too) which is favourable in a complex system such as Eq. (1). After this cancelation, pole placement will be used; and the most important feature is (as stated in Section 1.2) that it will be used in input-output decoupled form.

\subsection{Control in Lie Algebra Form}

Starting from the estimated system, after some rearrangement, the system becomes suitable for linearization:

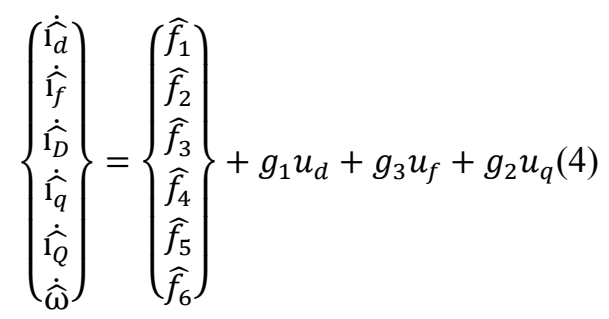

The $\widehat{f}_{1}, \widehat{f}_{2}, \ldots, \widehat{f}_{6}$ are parts of estimated states (3) as given in Eq. (4).

Excitation (field) voltage will not be controlled, but will remain a constant. So the system (4) will be a bit simplified.

Control will make a tracking system of two outputs: rotor speed, and square of stator magnetic flux:

$$
\hat{h}_{1}=\widehat{\omega} ; \hat{h}_{2}=\widehat{\varphi}_{d}^{2}+\widehat{\varphi}_{q}^{2}
$$

It is necessary to separate the first output into two variables; so the new one $\hat{h}_{11}$ (an electric torque) is introduced. According to Lie algebra, output differentials are made:

$$
\begin{gathered}
\dot{\hat{h}}_{1}=\mathrm{L}_{\hat{f}} \widehat{h}_{1}=\mathrm{j}_{1} \widehat{\iota}_{d} \widehat{l}_{q}+j_{2} \widehat{l}_{f} \widehat{l}_{q}+j_{3} \widehat{\iota}_{q} \widehat{l}_{D}+j_{4} \widehat{l}_{d} \widehat{l}_{Q}+j_{5} M_{T^{+}} \\
+k_{61} e_{1}+k_{62} e_{2}+k_{64} e_{4}+k_{66} e_{6}
\end{gathered}
$$

Or:

$$
\dot{\hat{h}}_{1}=\hat{h}_{11}+g_{5} M_{T}+\Delta
$$

Where,

$$
\begin{gathered}
\hat{\mathrm{h}}_{11}=j_{1} \widehat{\iota_{d}} \widehat{\iota}_{q}+j_{2} \widehat{\iota_{f}} \widehat{\iota_{q}}+j_{3} \widehat{\iota_{q}} \widehat{\iota_{D}}+j_{4} \widehat{\iota_{d}} \widehat{\iota_{Q}} \\
\Delta=k_{61} e_{1}+k_{62} e_{2}+k_{64} e_{4}+k_{66} e_{6}
\end{gathered}
$$

Recall the Lie derivative of $h$ along the vector field $f$ defined by:

$$
\mathrm{L}_{\mathrm{f}} \mathrm{h}=\frac{\partial h}{\partial x} f(x)
$$

Differentialof $\hat{h}_{11}$ in Lie algebra form is:

$$
\dot{\hat{h}}_{11}=\mathrm{L}_{\hat{f}} \hat{h}_{11}+\mathrm{L}_{\mathrm{g} 1} \hat{h}_{11} \mathrm{u}_{d}+\mathrm{L}_{\mathrm{g} 2} \hat{h}_{11} \mathrm{u}_{q}
$$

Where,

$$
\begin{aligned}
& g_{1}=\left[\begin{array}{llllll}
a_{6} & b_{6} & c_{6} & 0 & 0 & 0
\end{array}\right], \\
& g_{2}=\left[\begin{array}{lllllll}
0 & 0 & 0 & d_{6} & f_{6} & 0
\end{array}\right]
\end{aligned}
$$

Similarly, for the second output:

$$
\dot{\hat{h}}_{2}=\mathrm{L}_{\hat{f}} \hat{h}_{2}+\mathrm{L}_{\mathrm{g} 1} \hat{h}_{2} \mathrm{u}_{d}+\mathrm{L}_{\mathrm{g} 2} \hat{h}_{2} \mathrm{u}_{q}
$$

The system of estimated outputs is now:

$$
\left[\begin{array}{c}
\dot{\overline{\mathrm{h}_{1}}} \\
\dot{\widehat{\mathrm{h}_{11}}} \\
\dot{\widehat{\mathrm{h}_{2}}}
\end{array}\right]=\left[\begin{array}{c}
\hat{h}_{11}+g_{5} M_{T}+\Delta \\
\mathrm{L}_{\hat{f}} \hat{h}_{11}+\mathrm{L}_{\mathrm{g} 1} \hat{h}_{11} \mathrm{u}_{d}+\mathrm{L}_{\mathrm{g} 2} \hat{h}_{11} \mathrm{u}_{q} \\
\mathrm{~L}_{\hat{f}} \hat{h}_{2}+\mathrm{L}_{\mathrm{g} 1} \hat{h}_{2} \mathrm{u}_{d}+\mathrm{L}_{\mathrm{g} 2} \hat{h}_{2} u_{q}
\end{array}\right] \text { (13) }
$$

And the last two can be directly controlled:

$$
\left[\begin{array}{c}
\dot{\mathrm{h}_{11}} \\
\dot{\mathrm{h}_{2}}
\end{array}\right]=\left[\begin{array}{c}
\mathrm{L}_{\hat{f}} \hat{h}_{11} \\
\mathrm{~L}_{\hat{f}} \hat{h}_{2}
\end{array}\right]+G\left[\begin{array}{l}
\mathrm{u}_{d} \\
\mathrm{u}_{q}
\end{array}\right]
$$

where, $G$ is decoupling matrix:

$$
G=\left[\begin{array}{ll}
\mathrm{L}_{\mathrm{g} 1} \hat{h}_{11} & \mathrm{~L}_{\mathrm{g} 2} \hat{h}_{11} \\
\mathrm{~L}_{\mathrm{g} 1} \hat{h}_{2} & \mathrm{~L}_{\mathrm{g} 2} \hat{h}_{2}
\end{array}\right]
$$

Matrix $G$ is nonsingular in any operating point, except in the origin. Singularity in the origin can be easily avoided by putting initial conditions of the state observer a bit different from zero.

The control law is defined as follows:

$$
\left[\begin{array}{l}
\mathrm{u}_{d} \\
\mathrm{u}_{q}
\end{array}\right]=G^{-1}\left[\begin{array}{c}
-\mathrm{L}_{\hat{f}} \hat{h}_{11}-k_{p 1} e_{8}+\dot{h}_{11 r e f}-e_{7} \\
-\mathrm{L}_{\hat{f}} \hat{h}_{2}-k_{p 2} e_{9}+\dot{h}_{2 r e f}
\end{array}\right]
$$

where, Eq. (16) shows input-output decoupled control of speed (torque) and flux.

With errors $\quad \mathrm{e}_{7}=\hat{h}_{1}-h_{1 \text { ref }} \quad ; \quad \mathrm{e}_{8}=\hat{h}_{11}-$ $h_{11 \text { ref }} ; \mathrm{e}_{9}=\hat{h}_{2}-h_{2 \text { ref }}$ and $h_{11 \text { ref }}$ defined as: 
$h_{11 r e f}=\dot{h}_{1 r e f}-g_{5} M_{T}-k_{p 0} e_{7}-\Delta$, output error dynamics is gained:

$$
\left\{\begin{array}{l}
\dot{\mathrm{e}}_{7} \\
\dot{\mathrm{e}}_{8} \\
\dot{\mathrm{e}}_{9}
\end{array}\right\}=\left\{\begin{array}{c}
e_{8}-k_{p 0} e_{7} \\
-k_{p 1} e_{8}-e_{7} \\
-k_{p 2} e_{9}
\end{array}\right\}
$$

\section{Internal Dynamics}

The relative degree of the system is lower than the system order, so it is necessary to check the systems internal dynamics.

There is a well known theorem of bounded function which states that the sum and product of bounded functions is also a bounded function. The reverse is also valid.

In this system, the second output (that is of course bounded by the reference) is sum and product composition of state variables:

$$
h_{2}=\left(L_{d} i_{d}+L_{m d} i_{f}+L_{m d} i_{D}\right)^{2}+\left(L_{q} i_{q}+L_{m d} i_{Q}\right)^{2}(18)
$$

Because the first output $h_{l}$ is $\omega$ all state variables are included, and it can be concluded that all internal dynamics are bounded.

\section{System Stability}

Lyapunov function of the entire system "observer + controller" is:

$$
V=V_{1}+V_{2}
$$

Here $V_{l}$ is Lyapunov observer function defined inEq. (3). If $V_{2}$ is defined as:

$$
V_{2}=\frac{e_{7}^{2}}{2}+\frac{e_{8}^{2}}{2}+\frac{e_{9}^{2}}{2}
$$

Its differential is:

$$
\dot{V}_{2}=e_{7} \dot{e}_{7}+e_{8} \dot{e}_{8}+e_{9} \dot{e}_{9}
$$

That is always negative if $k_{p 0}, k_{p 1}$ and $k_{p 2}$ are positive values.

Both functions $V_{1}$ and $V_{2}$ are Lyapunov stable, and it is concluded that the entire system is asymptotically stable.

Because it is not possible to prove that matrix $G$ is globally invertible, global asymptotic stability cannot be claimed.

\section{Control Scheme}

Previous considerations are outlined in the control scheme shown in Fig. 1.

Control will optimize the SM currents so that the difference (error) between estimated and reference trajectories is minimized.

It is necessary to have current and voltage measurements, rotor speed measurement and to know the load torque (if it is not constant). Measurement of rotor angle is also necessary, especially because of initial angle determination.

\section{Space Vector Modulation}

Modulation of voltage source inverter control signals is done by PWM (pulse width modulation). Nowadays, SVPWM (space vector pulse width modulation) is standard power converters switching technique.

Its advantages over other PWM techniques are: It has common modulator for all three phases, so interaction between phases is considered; it generates less harmonic distortion in both output voltage and current; it has more efficient use of supply voltage; and it has a constant switching frequency.

There are many SVPWM switching patterns. Because of a low THD (total harmonic distortion) over the whole modulation range, a symetrical pattern is chosen.

Higher inverter operating frequency increases precision of the control and the quality of current waveforms. In the following simulations the carrier frequency of about $6 \mathrm{kHz}$ was chosen; which is well within the operating range of modern power electronics. To build SVPWM it was neccesary to model some functions. As found in other SVPWM related literature, they are omitted from this paper.

\section{Case Study}

The complete simulation study for one ordinary SM is given here.

\subsection{Energetic Part}

Synchronous motor will not be directly connected to inverter; to achieve the sinusoidal waveforms of 


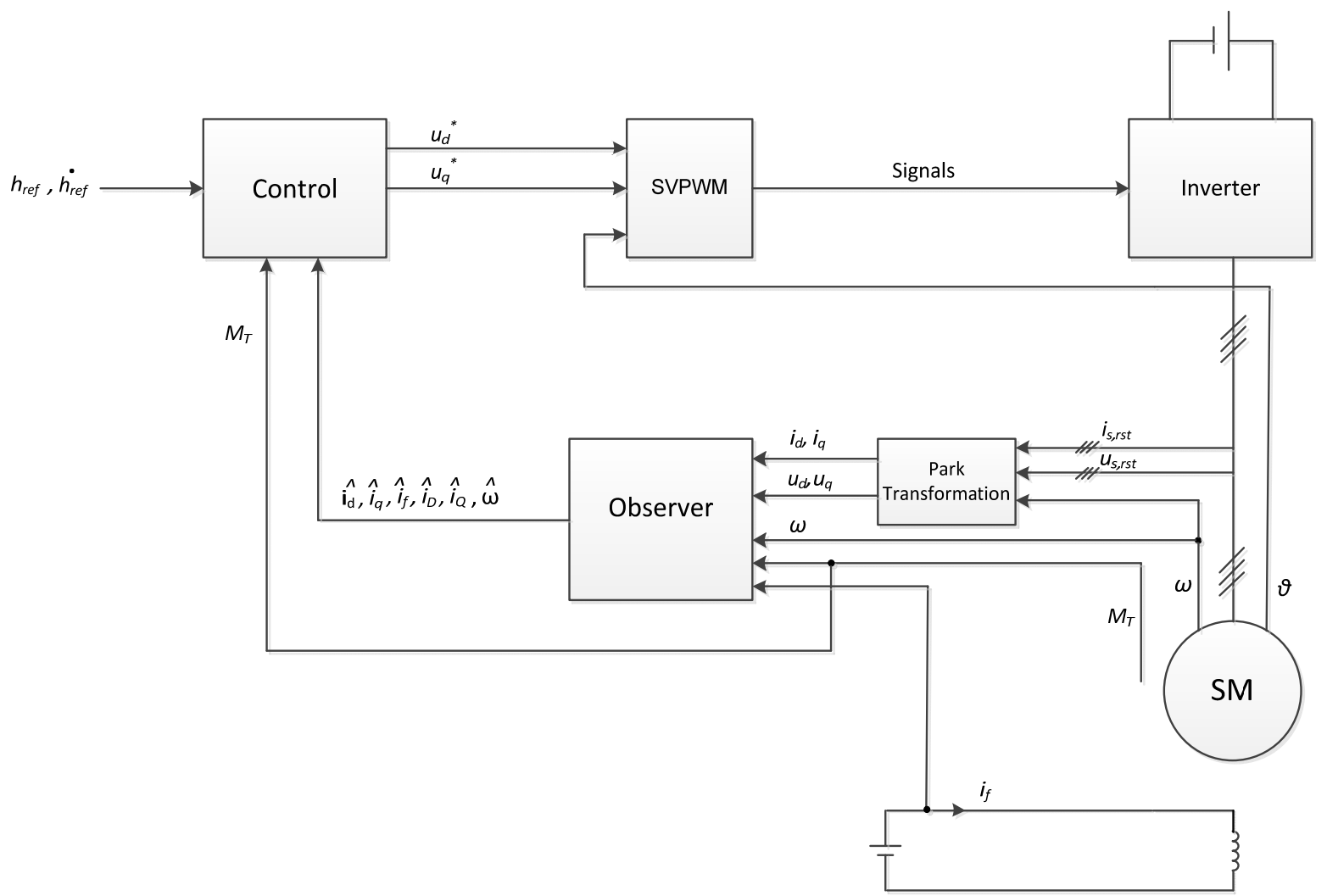

Fig. 1 Control scheme.

current and voltage it will be necessary to connect the filter: reactor in series, and the capacitor (with series resistor) in parallel to the SM.

Now, it is necessary to go back to the system equations and to take reactor inductance into consideration. Due to the high impedance of parallel connected capacitor (with resistor), its influence can be disregarded.

After some algebra this initial system is achieved:

$$
\left\{\begin{array}{l}
u_{d} \\
u_{q}
\end{array}\right\}=\left\{\begin{array}{l}
i_{d} R_{s}+\frac{d \psi_{d}}{d t}-\omega\left(\psi_{q}+i_{q} L_{\text {react }}\right) \\
i_{q} R_{s}+\frac{d \psi_{q}}{d t}+\omega\left(\psi_{d}+i_{d} L_{\text {react }}\right)
\end{array}\right\}
$$

It is now necessary to modify Eq. (1) on the basis of Eq. (22) and to include all necessary SM parameters to gain the form used in the simulation. The complete simulation model is given in Fig. 2.

The system is usually described in per unit system values, and so it will be used in this case, too.

Nominal parameters of SM are given as per unit values on SM stator basis; it will be necessary to calculate excitation voltage and reactor inductivity on the same basis.

SM nominal values: $S_{n}=8.1 \mathrm{kVA}, U_{n}=400 \mathrm{~V}$, $f_{n}=50 \mathrm{~Hz}, p=2, H=0.1406 \mathrm{~s}$.

Stator winding (p.u.): $R_{s}=0.082, L_{\sigma}=0.072, L_{m d}=$ $1.728, L_{m q}=0.823$.

Excitation winding (p.u.): $R_{f}=0.0612, L_{\sigma f}=0.18$.

Damper winding (p.u.): $R_{k d}=0.159, L_{\sigma k d}=0.117, R_{k}$ $=0.242, L_{\sigma k q}=0.162$.

Reactor (p.u.): $L_{\text {react }}=0.158$.

Some standard values of RLC filter are used in this work:

Inductance: $10 \mathrm{mH}$;

Capacitance: $4 \mathrm{nF}$;

Resistance: $10 \mathrm{k} \Omega$.

\subsection{Observer Simulation}

For the given SM parameters and Lyapunov function, convergence coefficients have to be found.

They are: $k_{3}=2 ; k_{2}=-0.2$; 


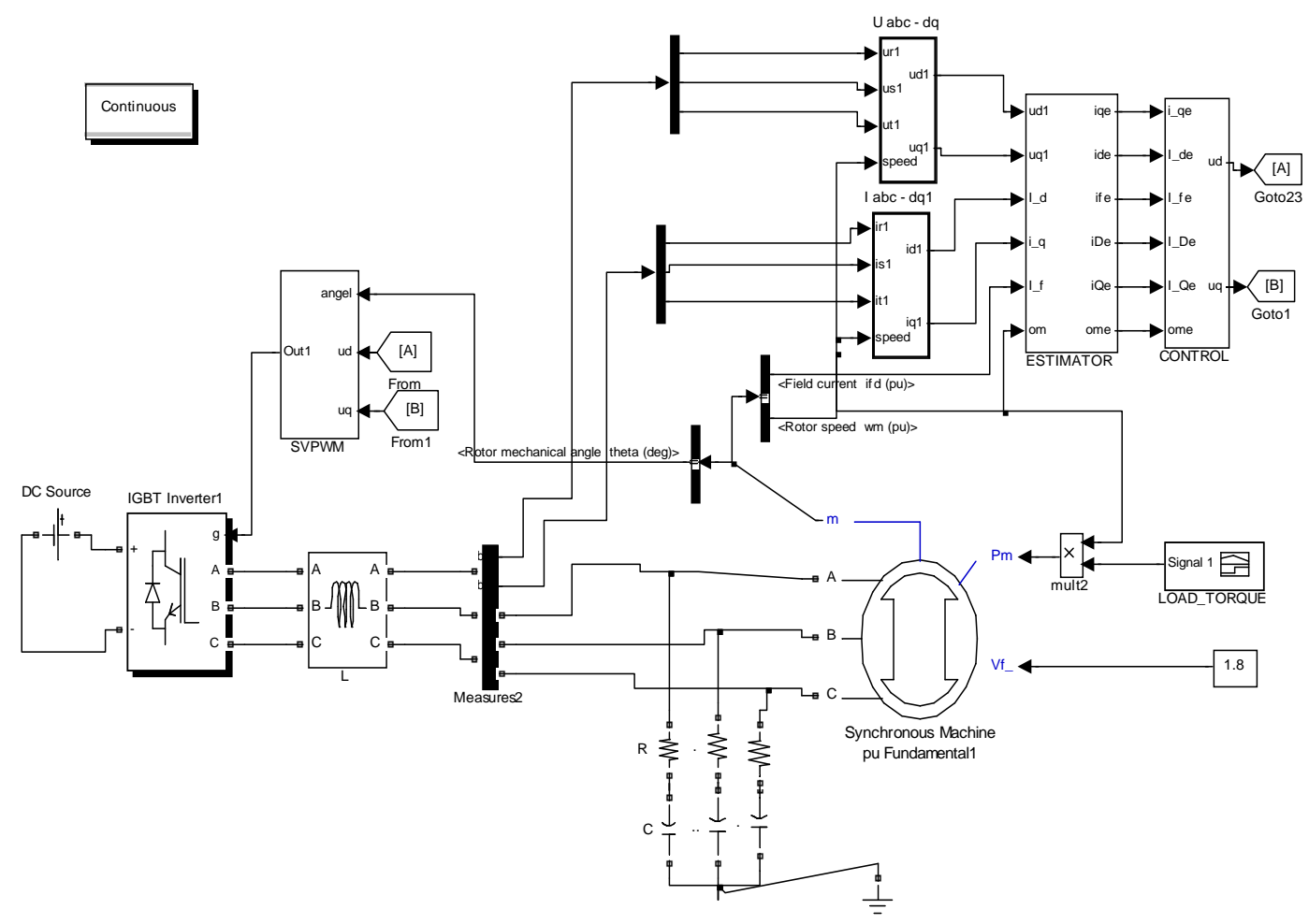

Fig. 2 Simulation model.

$$
\begin{gathered}
k_{1}=\frac{0.062+0.1585 \sqrt{0.156+182 \omega^{2}}}{\omega} \\
k_{32}=0.108+\frac{0.287}{k_{1}^{2}}-\frac{0.207 \omega}{k_{1}} \\
k_{52}=\frac{0.03}{k_{1}}-\omega+7 \widehat{\omega} \\
k_{34}=\frac{0.1}{k_{1}}-\frac{8.5 \omega}{k_{1}^{2}}-3.5 \widehat{\omega} \\
k_{51}=\frac{0.06}{k_{1}}+2.74 \omega+7.3 \widehat{\omega} \\
k_{36}=-3.2 \hat{\imath}_{Q}+i_{q}\left(-3.5+\frac{6.3}{k_{1}^{2}}\right)+\frac{0.6 i_{d}}{k_{1}} \\
k_{11}=50 ; k_{31}=\frac{0.6+0.3 k_{1}^{2}+0.5 k_{1} \omega}{k_{1}^{2}} \\
k_{56}=10.4 i_{d}+7 \hat{\imath}_{D}+7 i_{f} \frac{0.6 i_{q}}{k_{1}} \\
k_{12}=0.36 ; k_{14}=-2.78 \widehat{\omega} \\
k_{16}=6 i_{q}+3.2 \hat{\imath}_{q}+11.6 \hat{\imath}_{Q} \\
-2.25 i_{q}+6.2 \hat{\imath}_{q}-2.1 \hat{\imath}_{Q} \\
k_{46}=-5 i_{d}-2.2 \hat{\imath}_{D}-2.2 i_{f} \\
k_{44}=55 ; k_{66}=65 ; \\
=54
\end{gathered}
$$

$$
k_{21}=k_{41}=k_{61}=k_{62}=k_{64}=0
$$

A stable observer is thus completed and its simulation is done. Observed states' errors, as well as damper winding observed currents are shown. Given results are for the SM starting up to 0.3 p.u. rotor speed. In Figs. 3, 4 and 7, the current observer errors for currents $i_{d}, i_{f}, i_{q}$ are given respectively. As they are known states; their errors are (according to expectation) approximately zero. In Figs. 5 and 8, the damper winding observed currents iD and iQ are given; in Figs. 6 and 9 their respective errors are also shown. Because of high performance control demand and the field winding that is initially supplied with nominal voltage; the damper winding currents rapidly rises at the start and the observer is good enough to follow the changes. In Fig. 10, rotor speed observed error is given.

\subsection{Starting up to 0.3 p.u. Rotor Speed}

Matlab Simulink simulation of the following characteristics is made: continuous system, variable step integration, relative tolerance 1e-3, absolute tolerance 1e-4. 


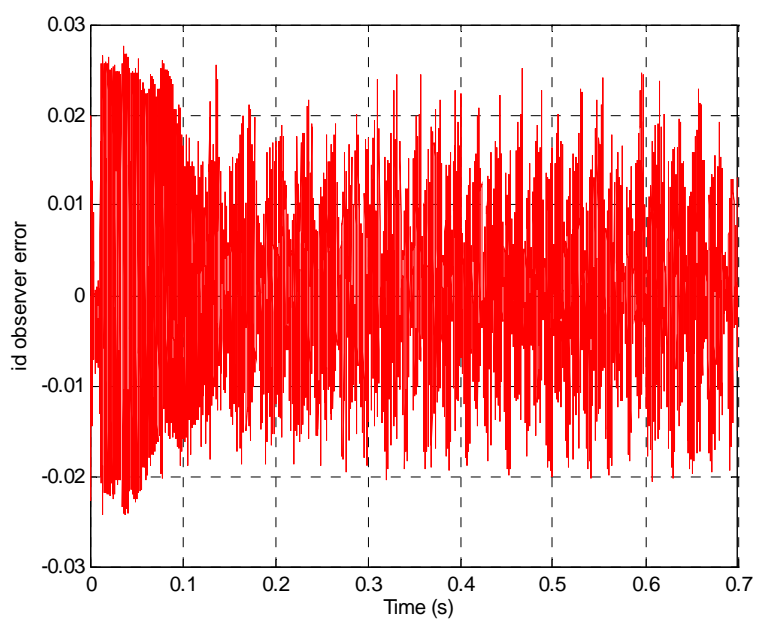

Fig. $3 i_{d}$ current observer-error.

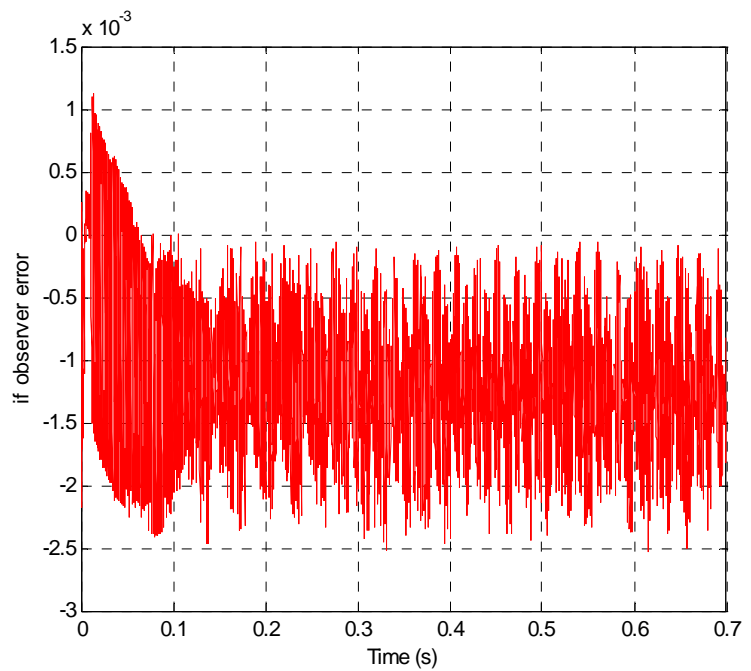

Fig. $4 i_{f}$ current observer-error.

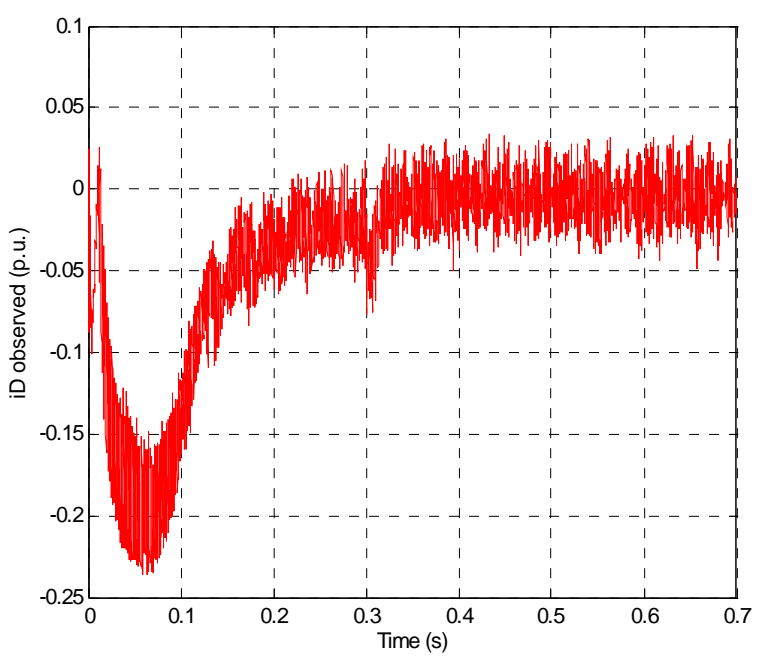

Fig. 5 Damper d-axis observed current.

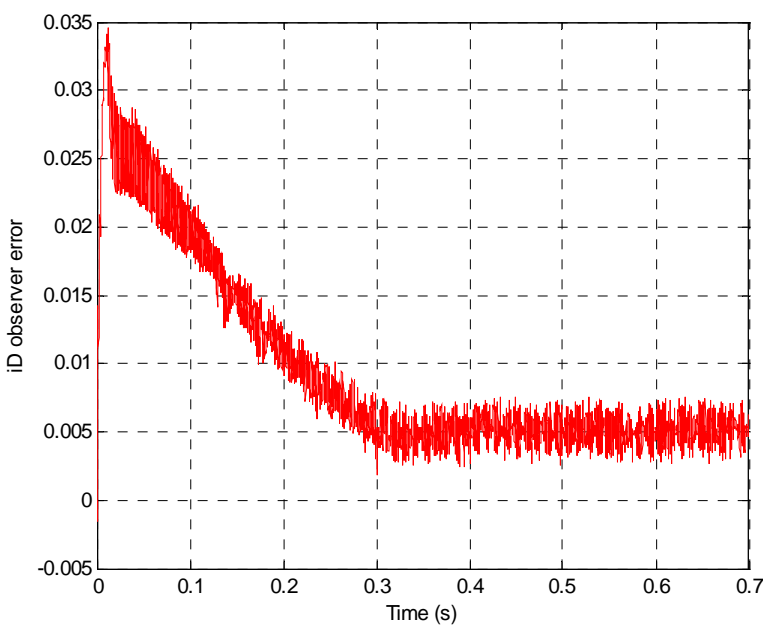

Fig. 6 Damper d-axis observed current-error.

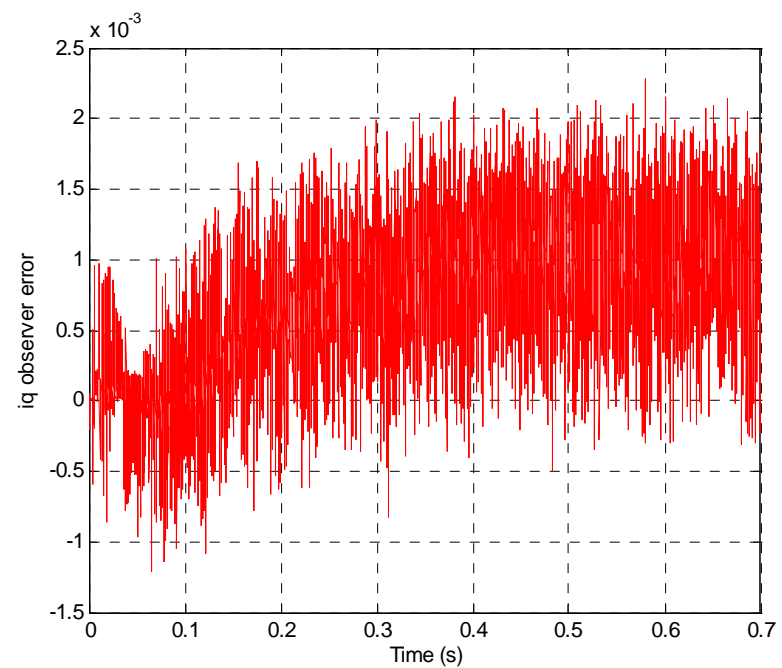

Fig. $7 \quad i_{q}$ current observer-error.

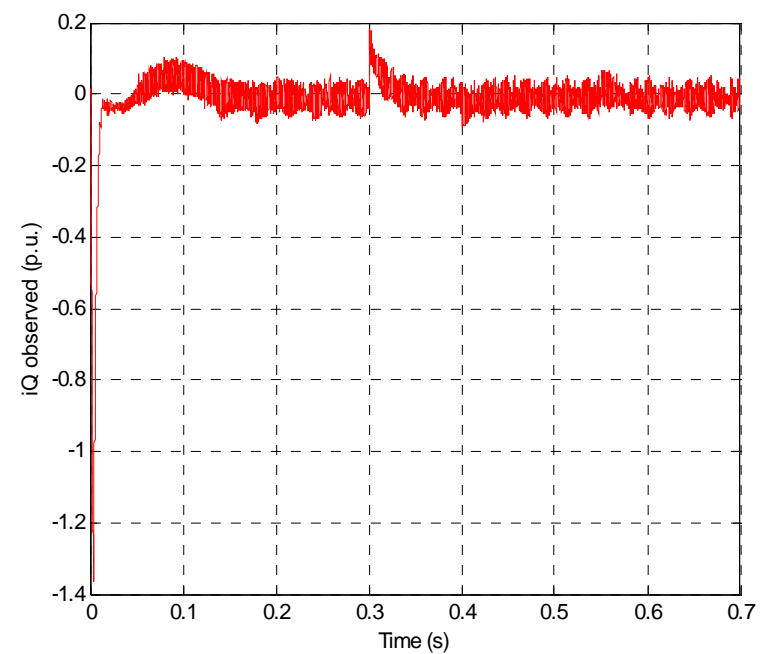

Fig. 8 Damper q-axis observed current. 


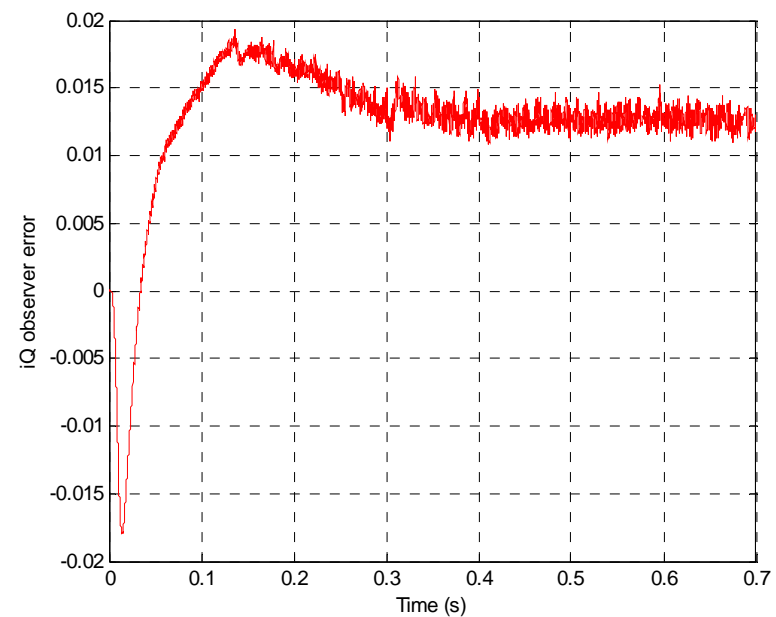

Fig. 9 Damper q-axis observed current-error.

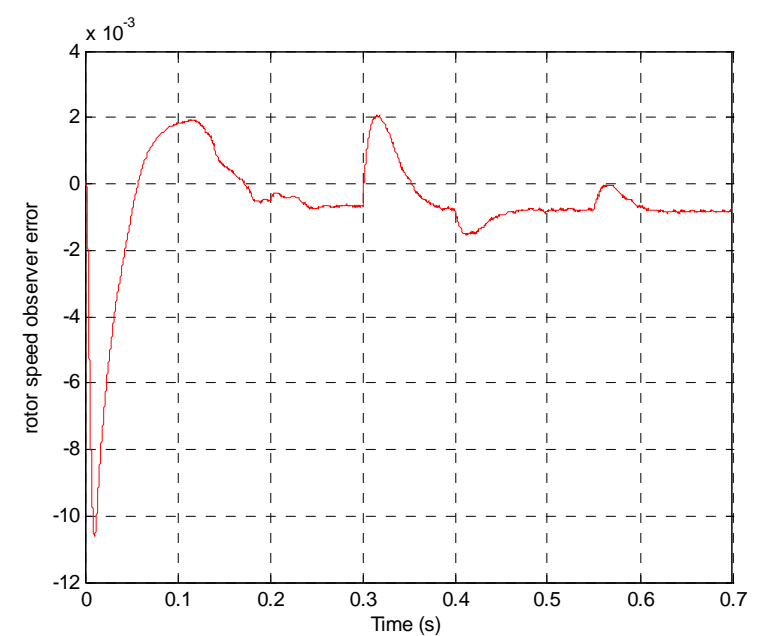

Fig. 10 Rotor speed observed-error.

Figures show SM starting: rotor speed (Fig. 11), stator currents (Figs. 12 and 13) and voltages (Figs. 14 and 15), rotor (field) current (Fig. 16), electromagnetic torque (Fig. 17), load torque (Fig. 18), stator magnetic flux (Fig. 19), speed and flux errors (Figs. 20 and 21). Both references, as well as load torque increase linearly until SM reaches the required speed. The results show a successful starting with certain errors primarily in the beginning, while the stationary error is about $1 \%$.

The selection of a stationary speed of 0.3 p.u. was done solely for practical reasons (saving time, lack of computer memory). In addition to the startup, step change in load torque in the steady state is done. The rotor speed error during step increase reaches up to about $1.5 \%$.

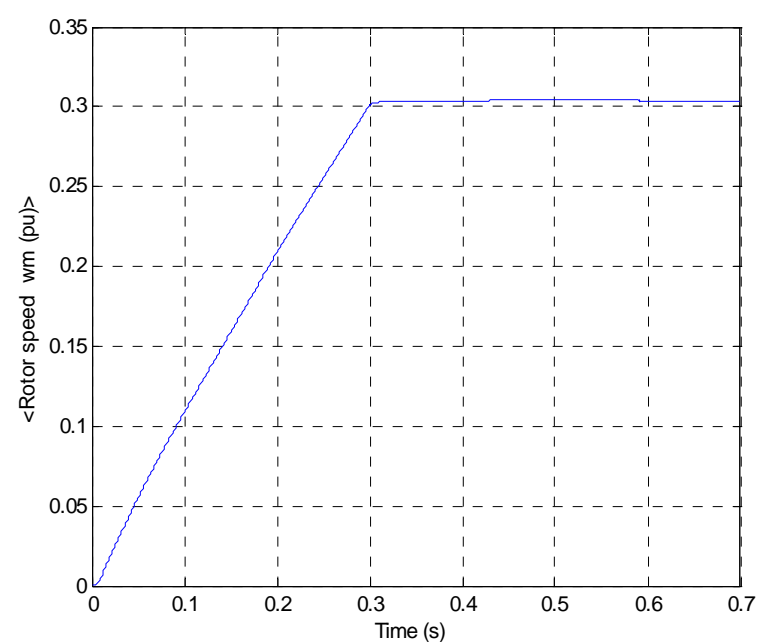

Fig. 11 Rotor speed.

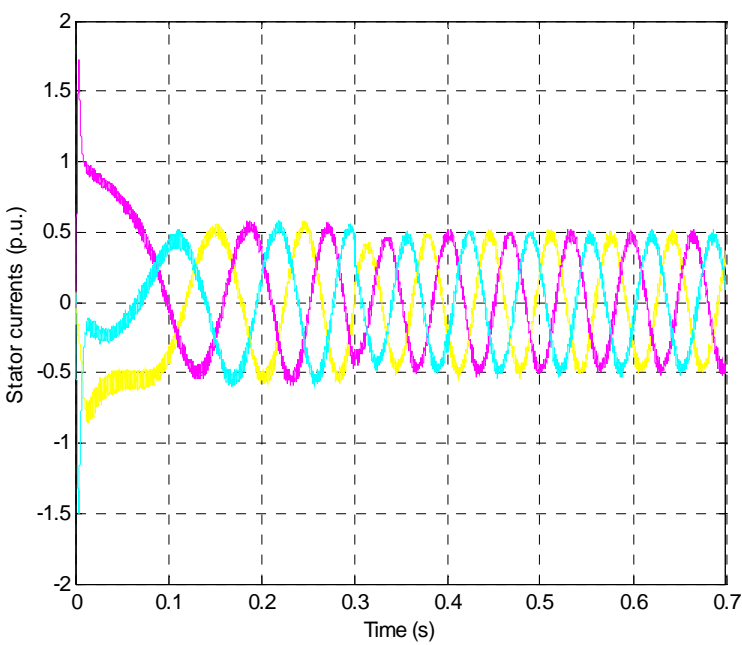

Fig. 12 Stator currents.

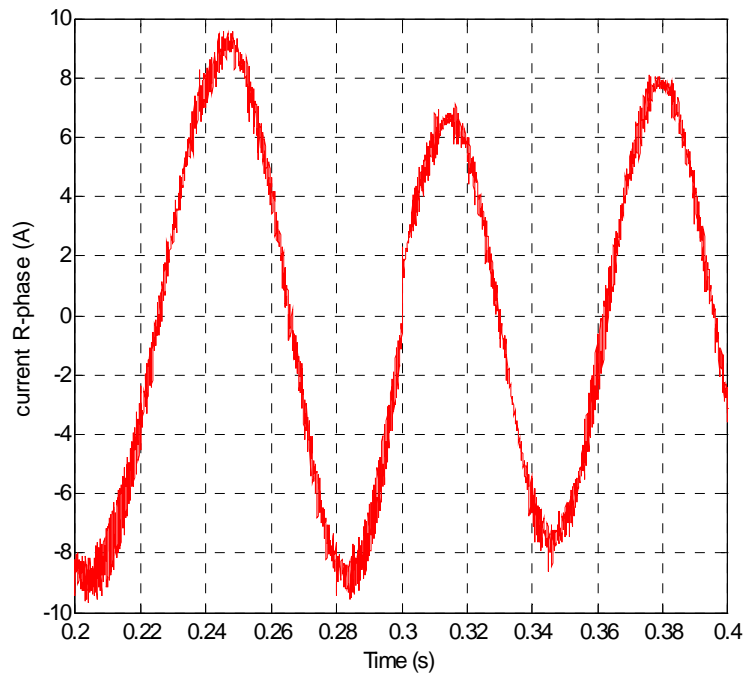

Fig. 13 R-phase current. 


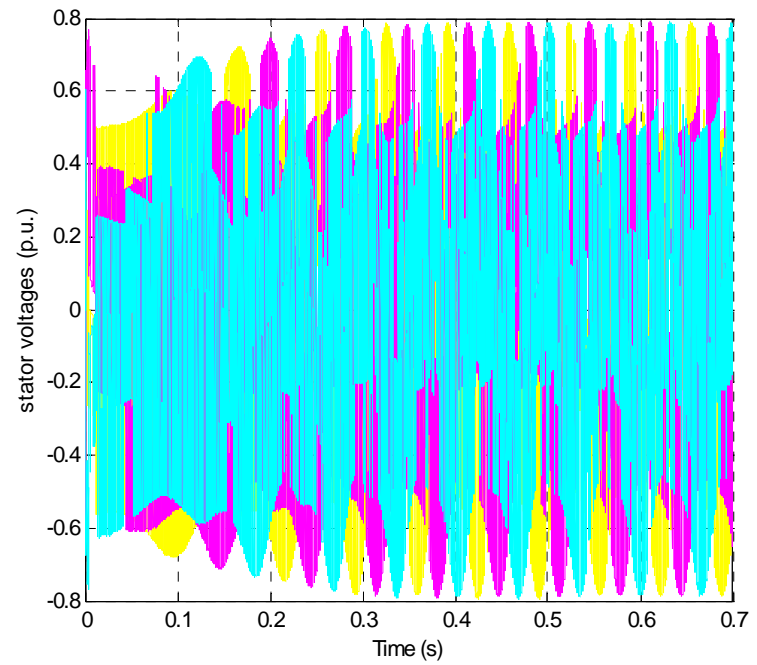

Fig. 14 Stator voltages.

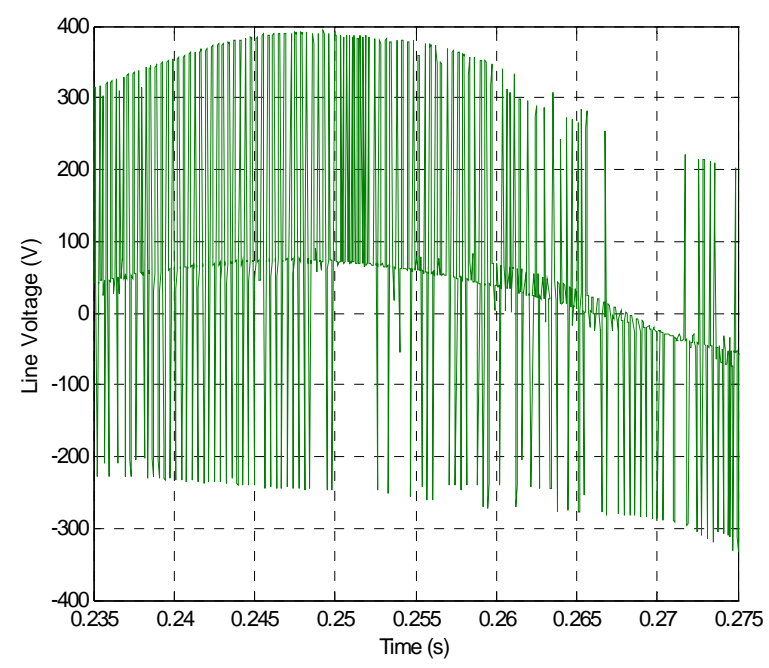

Fig. 15 Line voltage.

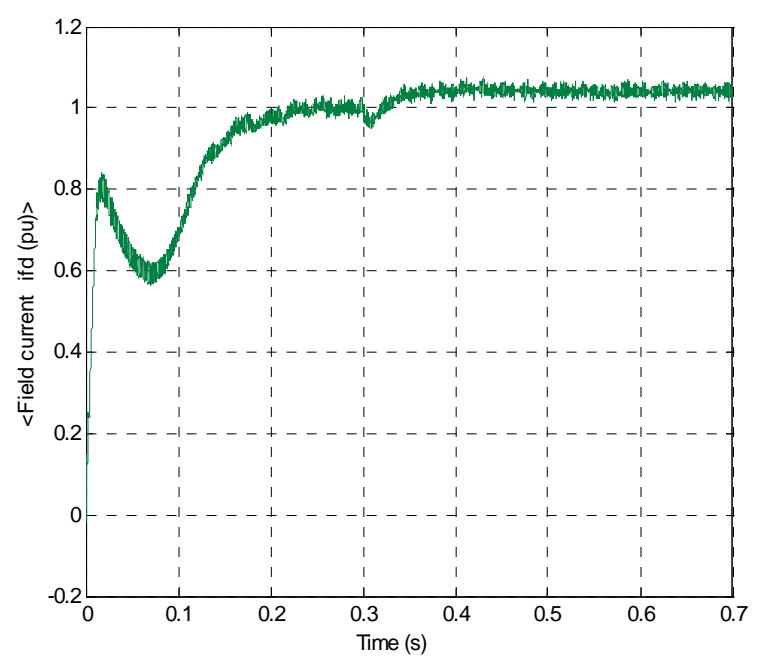

Fig. 16 Rotor current.

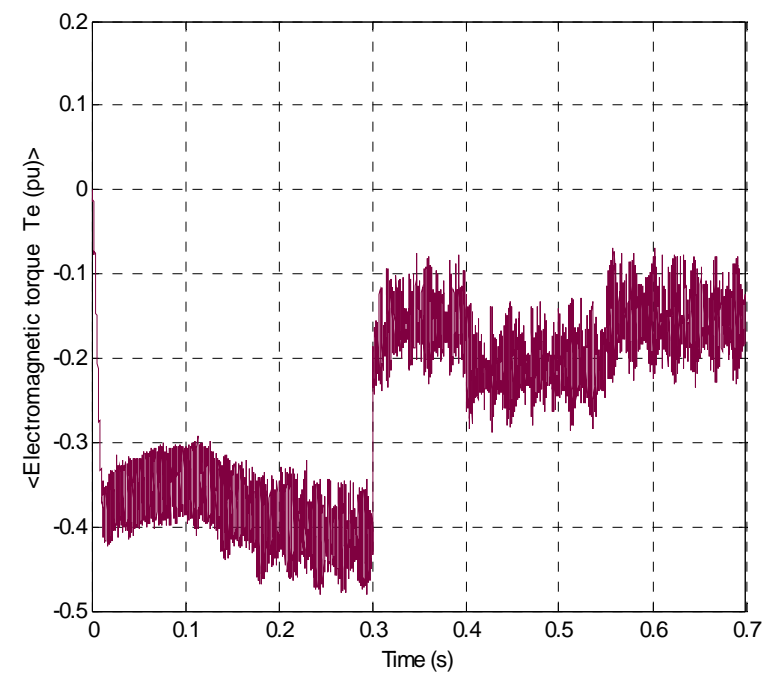

Fig. 17 Electromagnetic torque.

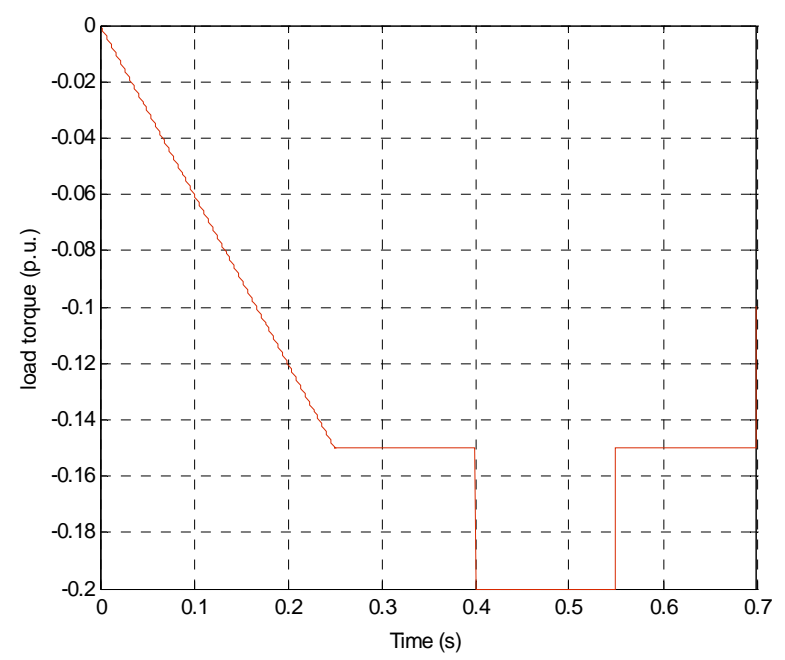

Fig. 18 Load torque.

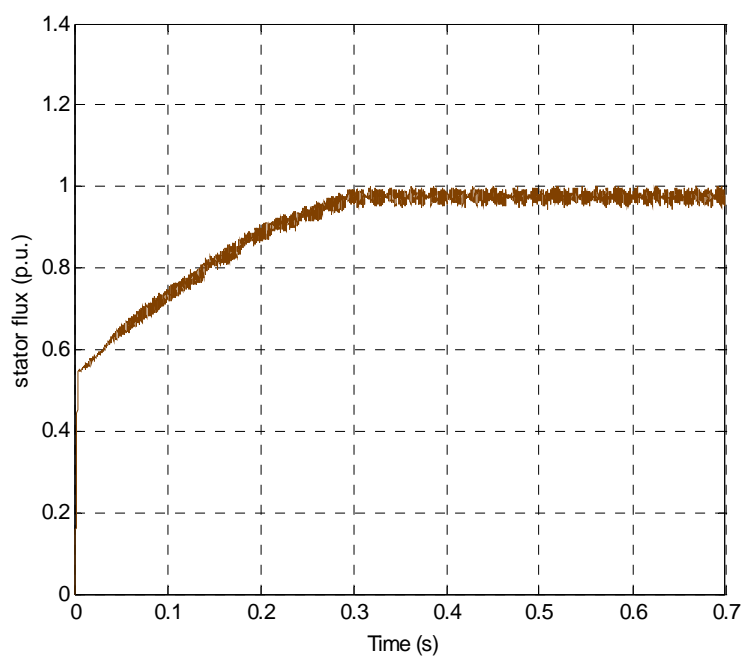

Fig. 19 Stator flux. 


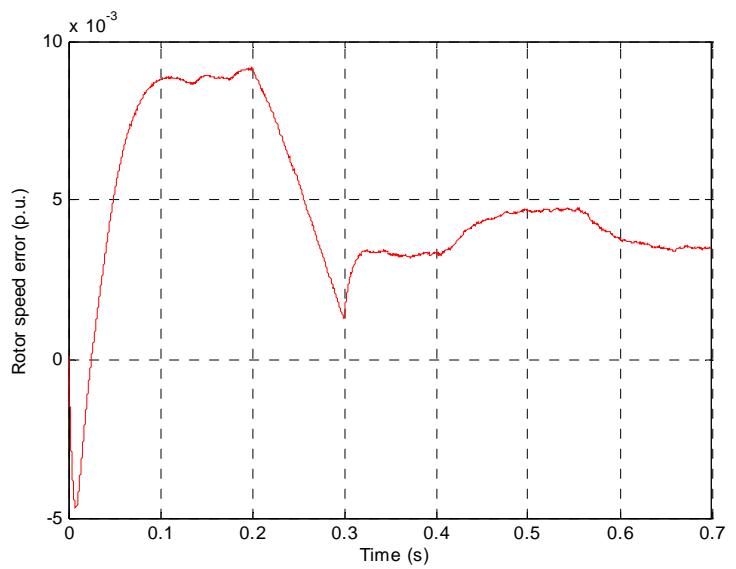

Fig. 20 Rotor speed-error.

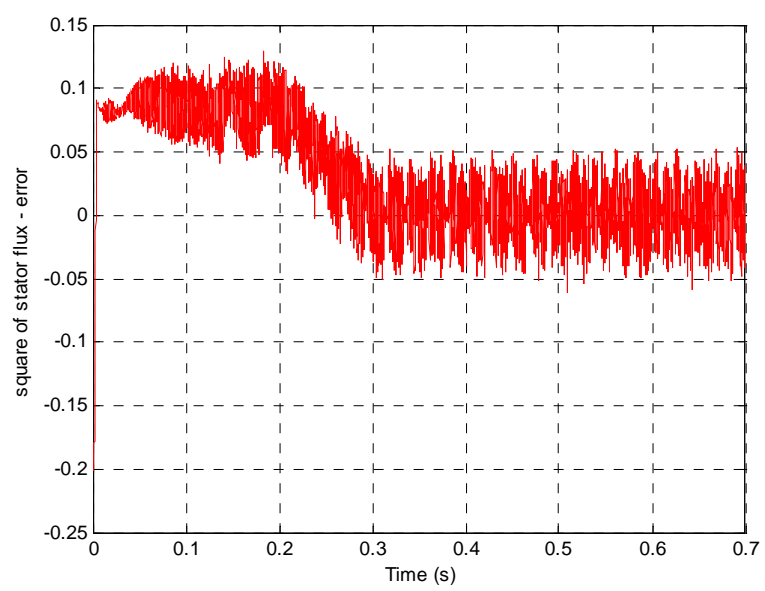

Fig. 21 Square of stator flux-error.

\subsection{Starting up to Nominal Rotor Speed}

In the second simulation, rotor speed reaches its nominal value. But, because of already mentioned computing restrictions, it was necessary to reduce the precision (relative tolerance $7 \mathrm{e}-3$, absolute tolerance 7e-4). Figs. 22 and 23 show rotor speed and stator flux. Output reference errors are given in Fig 24. (rotor speed) and Fig 25. (square of stator flux). Good control is achieved as in the first simulation.

\section{Contribution}

A novel method for control of synchronous motors with excitation and damper windings is presented. The method is based on the feedback linearization principle.

It is necessary to begin with a description of the synchronous motor using currents as the state variables. After that, the output variables are defined

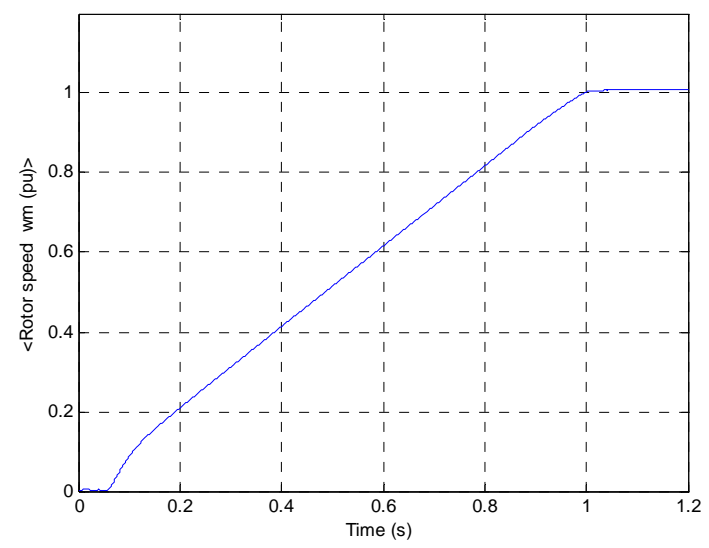

Fig. 22 Rotor speed.

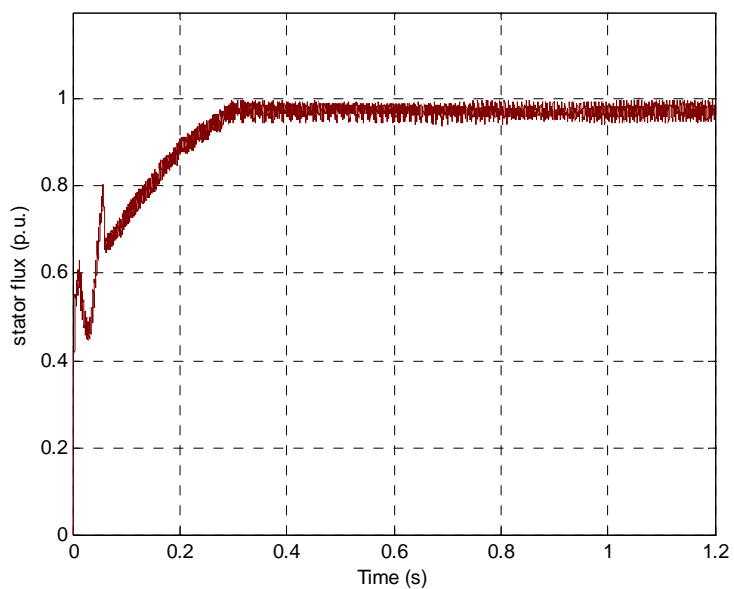

Fig. 23 Stator flux.

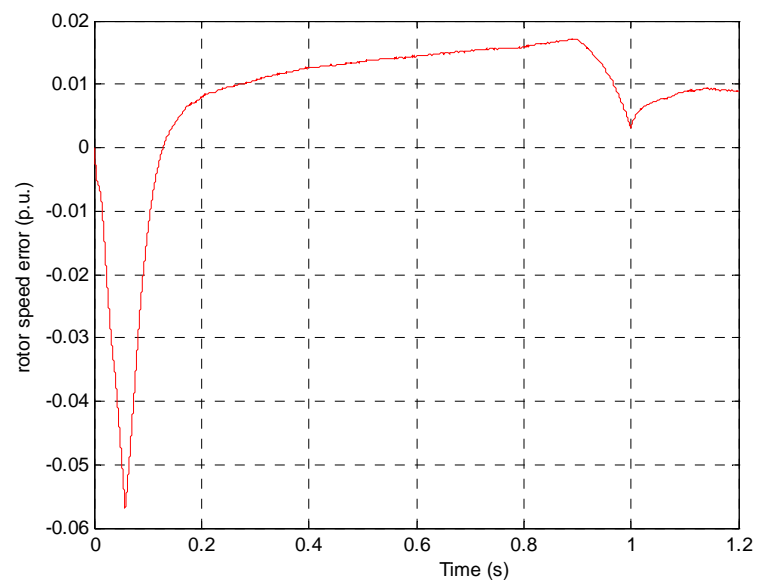

Fig. 24 Rotor speed-error.

and accordingly mathematically provable stable system is established. For the implementation of the control in terms of synchronous motor electric drive, it is necessary to apply modulation for power converter switching. For that purpose space vector pulse width 


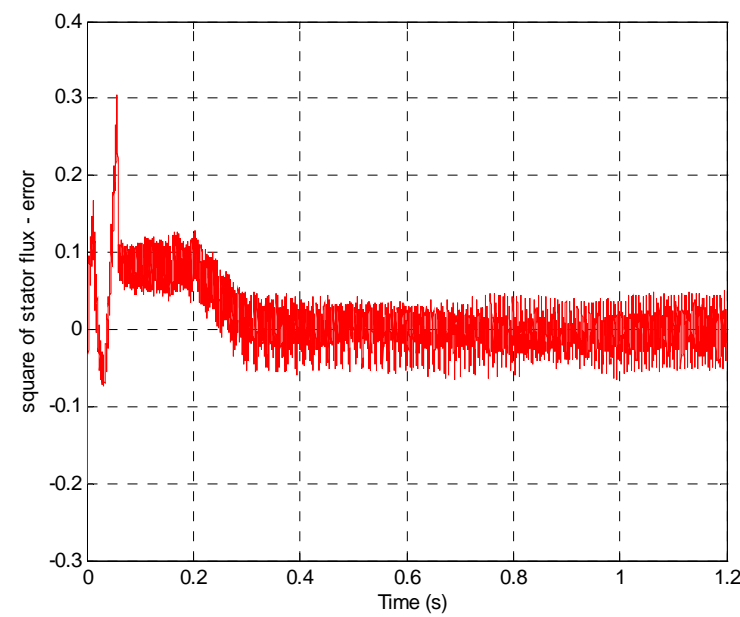

Fig. 25 Square of stator flux-error.

modulation SVPWM is used. In addition, for obtaining quality waveforms of stator voltage and current, it is necessary to define an appropriate filter.

Since synchronous machine model takes damper windings into consideration, it was necessary to develop an adequate estimation of the damper currents. Previous methods for estimation of damper currents are inadequate for this kind of dynamics. Therefore, well defined Lyapunov function enables high-quality estimation of total SM state variables, and can be applied to all SM.

\section{Future Work}

Controller is discretized in a way that switching frequency and sampling time have acceptable (realistic) values. Simulations of this sampled data system give similarly good results as for continuous system. In the future, adaptive algorithm for field and stator resistance will be included in the control system.

\section{Conclusions}

The paper presents the simulation of starting of the synchronous motor with excitation and damper windings. Simulations are performed with different values of steady state speed, considering different computation errors. In addition to the starting, simulation of load torque step change is done. Simulations give good results.
To achieve desired SM starting, tracking control system of two output variables is developed: one being rotor speed, and other square of stator flux. Excitation voltage is kept a constant value during the whole simulation.

\section{References}

[1] D. Beliaev, E. Ilyin, A. Shatokhin, A. Weinger, Synchronous drives with field oriented vector control and their industrial implementation, in: 13th European Conference on Power Eletronics and Applications, Barcelona, Sept. 2009.

[2] C. Szabo, M. Imecs, I.I. Incze, Vector control of the synchronous motor operating at unity power factor, in: 11th International Conference on Optimization of Electrical and Electronic Equipment, Brasov, May 2008, pp. 15-20.

[3] A. El Magri, F. Giri, A. Abouloifa, M. Haloua, Nonlinear control of wound-rotor synchronous-motor, in: International Conference on Control Applications, Munich, Germany, 2006, pp. 3110-3115.

[4] H. Xue, J. Jiang, Passivity-based control of synchronous motors, in: IEEE 6th International Power Electronics and Motion Control Conference, IPEMC, Wuhan, China, 2009, p. 2047.

[5] R. Marino, P. Tomei, C.M. Verreli, Nonlinear control for speed-sensorless synchronous motors with damping windings, in: International Conference on International Conference on Power Engineering, Energy and Electrical Drives, Setubal, Portugal, 2007, pp. 742-747.

[6] R. Marino, P. Tomei, C.M. Verreli, Nonlinear adaptive output feedback control of synchronous motors with damping windings, in: 32nd Annual Conference on IEEE Industrial Electronics, Paris, France, 2006, pp. 1131-1136.

[7] M. Flota, R. Alvarez-Salas, H. Miranda, E. Cabal-Yepez, R.J. Romero-Troncoso, Nonlinear observer-based control for an active rectifier, Journal of Scientific \& Industrial Research 70 (2011) 1017-1025.

[8] M. Chenafa, A. Mansouri, A. Bouhenna, E. Etien, A. Belaidi, M.A. Denai, Global stability of linearizing control with a new robust nonlinear observer of the induction motor, Journal of Appl. Math. Comput. Sci. 15 (2) (2005) 235-243.

[9] A. Merabet, Nonlinear model predictive control for induction motor drive, in: Frontiers of Model Predictive Control, 2012.

[10] P. Vaclavek, P. Blaha, Lyapunov-function-based flux and speed observer for AC induction motor sensorless control and parameter estimation, IEEE Trans. Ind. Electron 53 (1) (2006) 154-161. 